\title{
Identification of Sterols and Steroidal 3-Ones in the Seeds of Echinochloa frumentacea
}

\author{
Yasuhisa NARUMI*1, Chiharu GoTOH*1, Takahiro NoGUCHI*2, \\ Shozo FUjIOKA ${ }^{* 3}$, Takao YокотA ${ }^{* 4}$ and Suguru TAKATSUTO*1 \\ *1 Department of Chemistry, Joetsu University of Education \\ (1, Yamayashiki-machi, Joetsu-shi, Niigata-ken 943-8512) \\ *2 Tama Biochemical Co. Ltd. \\ (2-7-1, Nishishinjuku, Shinjuku-ku, Tokyo 163-0704) \\ *3 Plant Functions Laboratory, The Institute of Physical and Chemical Research (RIKEN) \\ (2-1, Hirosawa, Wako-shi, Saitama-ken 351-0198) \\ * 4 Department of Biosciences, Teikyo University \\ (1-1, Toyosatodai, Utsunomiya-shi, Tochigi-ken 320-8551)
}

\begin{abstract}
Sterols and steroidal 3-ones were detected in the seeds of Echinochloa frumentacea (Roxb.) Link. Twenty sterols were identified by direct GC-MS comparison with authentic samples and the compositions of both free and esterified sterol fractions were determined. Eight steroidal 3-ones were found present in the seeds.
\end{abstract}

Key words : Echinochloa frumentacea (Roxb.) Link, seed, sterol, steroidal 3-one

\section{Introduction}

Recently, much attention has been focused on stanols, not only because sitostanol and its fatty acid esters lower the serum cholesterol level by suppressing cholesterol absorption ${ }^{1,2)}$, but because campestanol is a precursor of a phytohormone brassinolide $^{3)}$. As an early part of brassinolide biosynthesis, it has recently been demonstrated that a series of biosynthetic reactions, campesterol $\rightarrow(24 R)$-24-methylcholest-4-en-3 $\beta$-ol $\rightarrow(24 R)$-24methylcholest-4-en-3-one $\rightarrow$ (24R)-24-methyl-5 $\alpha$ cholestan-3-one $\rightarrow$ campestanol, takes place in the shoots of Arabidopsis thaliana and the cultured cells of Catharanthus roseus ${ }^{4}$. It has analogously been proposed that sitostanol is also biosynthesized from sitosterol through $\mathrm{C}_{29}$ homologs by a pathway parallel to that of campestanol from campesterol in plants, based on the co-occurrence of the corresponding intermediates in the seeds of Cannabis sativa ${ }^{5,6)}$, wheat ${ }^{7)}$, and foxtail millet ${ }^{7}$. We have recently reported a high content of sitostanol in the milled grains and seeds of foxtail millet ${ }^{8), 9}$.

Corresponding author: Suguru TAKATSUTO

E-mail : takatuto@juen.ac.jp
Continuing our research program of sterols in cereal grains, we have now examined trace sterols and steroidal 3-ones in the seeds of Echinochloa frumentacea (Roxb.) Link, because of our preliminary results that cholesterol, 24-methylenecholesterol, and isofucosterol are present along with campesterol, campestanol, stigmasterol, sitosterol, and sitostanol. The seeds seems to be ideal to obtain basic information about the biosynthetic pathways of stanols, because of the co-occurrence of the sterols with six types of side chain. In this paper, we wish to report our identification results of sterols and steroidal 3-ones in E. frumentacea seeds.

\section{Experimental}

\subsection{Authentic Steroids}

The following authentic steroids used in this study (listed in Table $\mathbf{1}$ and 2 ) are available : sterol (1) was obtained from Wako, and sterols $(4,5,7$ $11,13-16$, and 18-20) are available as previously described $^{7,10), 11)}$, and steroidal 3-ones (23-28) are available as previously described ${ }^{7,12)}$. Sterols $(2,3$, 6, 12, and 17) and steroids 21 and 22 were synthesized $^{13)}$. 


\subsection{Extraction of a Lipid Fraction from the $\boldsymbol{E}$. frumentacea Seeds}

According to the reported method ${ }^{7)}$, the homogenized seeds of E. frumentacea $(3.0 \mathrm{~kg}$, harvested in China and the seeds were identified by Dr. Y. Iokawa of Joetsu University of Education) were extracted with a 1:1 mixture of chloroform and methanol and the extract was subjected to solvent partitioning to give a hexanesoluble lipid fraction $(155.84 \mathrm{~g})$.

$2 \cdot 3$ Separation of Fractions of Sterols from Steryl Esters, Steroidal 4-En-3-ones, $5 \alpha$-Steroidal 3-Ones, and Free Sterols

According to the reported method ${ }^{7}$, a sterol fraction A (1.27 g) derived from a steryl ester fraction, a $5 \alpha$-steroidal 3-one fraction B $(3.5 \mathrm{mg})$, a steroidal 4-en-3-one fraction C $(16.2 \mathrm{mg})$, and a free sterol fraction D $(1.29 \mathrm{~g})$ were obtained from the lipid fraction.

\subsection{Fractionation of Sterol Fractions A and} D

The sterol fraction A (300 mg) was acetylated in the usual manner and the resulting acetate was fractionated by $10 \% \mathrm{AgNO}_{3}$-silica gel column (2.0 $\mathrm{cm}$ i.d. $\times 32 \mathrm{~cm}$ ). Each fraction was collected in the amount of $10 \mathrm{~mL}$. The fractions $14 \sim 19$ with $1 \%$ diethyl ether $\left(\mathrm{Et}_{2} \mathrm{O}\right)$ /dichloromethane $\left(\mathrm{CH}_{2} \mathrm{Cl}_{2}\right)$ were collected as fraction A-1 $(21.1 \mathrm{mg})$, the fractions $20 \sim 23$ with $1 \% \mathrm{Et}_{2} \mathrm{O} / \mathrm{CH}_{2} \mathrm{Cl}_{2}$ as fraction A-2 $(128.4 \mathrm{mg})$, the fractions $24 \sim 31$ with $1 \%$ $\mathrm{Et}_{2} \mathrm{O} / \mathrm{CH}_{2} \mathrm{Cl}_{2}$ as fraction A-3 $(46.4 \mathrm{mg})$, the fractions $32 \sim 39$ with $1 \% \mathrm{Et}_{2} \mathrm{O} / \mathrm{CH}_{2} \mathrm{Cl}_{2}$ as fraction A-4 $(11.4 \mathrm{mg})$, the fractions $40 \sim 50$ with $3 \%$ $\mathrm{Et}_{2} \mathrm{O} / \mathrm{CH}_{2} \mathrm{Cl}_{2}$ as fraction $\mathrm{A}-5(4.8 \mathrm{mg})$, the fractions $51 \sim 60$ with ethyl acetate as fraction A-6 $(3.7 \mathrm{mg})$ and the fractions $61 \sim 70$ with ethyl acetate as fraction A-7 (76.8 mg).

Each fraction A-1 7 was saponified in the usual manner to provide, after the extractive work-up $\left(\mathrm{Et}_{2} \mathrm{O}\right)$, fraction A-1a (19.3 mg), A-2a (116.7 mg), A-3a (36.1 mg), A-4a (5.1 mg), A-5a (2.5 mg), A6a $(2.6 \mathrm{mg})$, and A-7a $(62.6 \mathrm{mg})$, respectively.

In the same manner, the sterol fraction D (300 mg) was acetylated, fractionated by $\mathrm{AgNO}_{3}$-silica gel column and saponified to give fraction $\mathrm{D}-1 \mathrm{a}$ (15.1 mg), D-2a (163 mg), D-3a (65 mg), D-4a (3 $\mathrm{mg})$, D-5a (1.1 mg), D-6a (27.7 mg), and D-7a (2.8 $\mathrm{mg})$.

\subsection{GC-MS Analysis}

GC-MS analysis was performed with a gas chromatograph-mass spectrometer (GC-MS QP5000, Shimadzu Corporation) equipped with a fused silica DB-1 capillary column $(30 \mathrm{~m} \times 0.25$ $\mathrm{mm}$ i.d., $0.25 \mu \mathrm{m}$ film thickness, J \& W Scientific), under the same condition as previously described $^{12)}$. Each sterol fraction was converted to trimethylsilyl (TMS) derivative and analyzed, whereas the steroidal 3-one and 4-en-3-one fractions were directly analyzed.

The relative retention times $\left(\mathrm{Rt}_{\mathrm{R}}\right)$ of each sterol TMS ether to cholesterol TMS ether were summarized in Table 1. Mass spectral data of the identified sterols were as follows. Cholestanol (2) TMS ether, $m / z$ (rel. int., \%) : $460\left(\mathrm{M}^{+}, 19\right), 445$ (35), 403 (13), 370 (24), 367 (15), 355 (37), 306 (27), 262 (14), 257 (10), 237 (8), 230 (18), 215 (100), 201 (20). Lathosterol (3) TMS ether, $\mathrm{m} / \mathrm{z}$ : $458\left(\mathrm{M}^{+}, 93\right), 443$ (36), 368 (10), 353 (35), 345 (14), 303 (11), 255 (100), 229 (34), 213 (58), 201 (13). 24-Methylenecholesterol (4) TMS ether, $\mathrm{m} / \mathrm{z}$ : $470\left(\mathrm{M}^{+}, 7\right), 455(5), 386(25), 380$ (14), $371(8)$, 365 (10), 343 (15), 341 (15), 296 (20), 281 (14), 257 (13), 255 (8), 253 (17), 243 (5), 213 (8), 129 (100). 24-Methyl-5 $\alpha$-cholest-24(28)-en-3 $\beta$-ol (6) TMS ether, $m / z: 388\left(\mathrm{M}^{+}-84,100\right), 373$ (34), 345 (54), 283 (26), 255 (49), 229 (23), 215 (36). Episterol (10) TMS ether, $\mathrm{m} / z: 470\left(\mathrm{M}^{+}, 7\right), 455(15), 386$ (21), 380 (5), 372 (8), 365 (12), 343 (100), 318 (4), 303 (4), 281 (9), 255 (16), 253 (22), 227 (21), 213 (28). 24-Ethylcholesta-5,25-dien-3 $\beta$-ol (12) TMS ether, $m / z: 484\left(\mathrm{M}^{+}, 20\right), 469(6), 394(20), 386$ (5), 379 (10), 371 (6), 355 (15), 343 (5), 296 (5), 281 (7), 255 (8), 253 (9), 227 (4), 213 (12), 129 (100). 24-Ethyl-5 $\alpha$-cholest-24(28)Z-en-3 $\beta$-ol (17) TMS ether, $m / z: 388\left(\mathrm{M}^{+}-98,100\right), 374$ (13), 345 (14), 305 (19), 283 (25), 255 (16), 229 (18), 215 (39), 201 (10). The mass spectral data of respective TMS ether of cholesterol (1), campesterol (5), campestanol (7), stigmasterol (8), $\Delta^{22}$-stigmastenol (9), 24-methyllathosterol (11), fucosterol (13), sitosterol (14), isofucosterol (15), sitostanol (16), 24-ethyldesmosterol (18), 24-ethyllathosterol (19), and avenasterol (20) were essentially the same as previously reported ${ }^{7,11), 12), 14)}$.

The $\mathrm{Rt}_{\mathrm{R}} \mathrm{S}$ of each steroidal 4-en-3-one to cholesterol TMS ether were summarized in Table 2. Mass spectral data of the identified steroidal 3ones were as follows. Cholest-4-en-3-one (21), $\mathrm{m} / \mathrm{z}$ (rel. int., \%) : $384\left(\mathrm{M}^{+}, 29\right), 342$ (14), 261 (27), 229 (33), 211 (9), 187 (14), 175 (9), 149 (33), 135 (19), 
124 (100). 24-Methylcholesta-4,24(28)-dien-3-one (22), $\mathrm{m} / \mathrm{z}: 396\left(\mathrm{M}^{+}, 15\right), 381$ (14), 313 (78), 312 (100), 297 (93), 281 (6), 269 (86), 256 (23), 245 (23), 243 (31), 231 (41), 229 (36), 227 (67), 209 (18), 187 (24), 173 (33), 161 (28), 149 (50), 147 (52), 135 (45), 133 (58), 124 (95). The mass spectral data of 24-methylcholest-4-en-3-one (23), 24-ethylcholesta-4,22E-dien-3-one (24), 24-ethylcholest-4-en-3-one (25), 24-ethylcholesta-4,24(28) $Z$-dien-3-one (26), 24-methyl-5 $\alpha$-cholestan-3-one (27), and 24-ethyl-5 $\alpha$-cholestan-3-one (28) were essentially the same as reported previously ${ }^{7,12}$.

\section{Results and Discussion}

As described in Experimental, a hexane-soluble lipid was obtained from the homogenized seeds of $E$. frumentacea. From this lipid, a sterol fraction $A$ of a steryl ester fraction, a steroidal 4-en-3-one fraction $\mathrm{B}$, a $5 \alpha$-steroidal 3-one fraction $\mathrm{C}$, and a free sterol fraction $\mathrm{D}$ were obtained.

The acetate of the sterol fraction $\mathrm{A}$ was fractionated by $10 \% \mathrm{AgNO}_{3}$-silica gel column. After converting to the TMS ether, each subfraction was analyzed by GC-MS. In the same manner,

Table 1 Sterol Composition of the Seeds of Echinochloa frumentacea.

\begin{tabular}{|c|c|c|c|}
\hline \multirow[b]{2}{*}{ Sterol (as a TMS ether) } & \multirow[b]{2}{*}{$\mathrm{Rt}_{\mathrm{R}}^{\mathrm{a})}$} & \multicolumn{2}{|c|}{ Composition (\%) } \\
\hline & & $\begin{array}{l}\text { Free sterol } \\
(430 \mathrm{mg} / \mathrm{kg})\end{array}$ & $\begin{array}{l}\text { Esterified sterol } \\
\quad(423 \mathrm{mg} / \mathrm{kg})\end{array}$ \\
\hline Cholesterol (1) & 1.000 & 1.21 & 4.64 \\
\hline Cholestanol (2) & 1.012 & 0.02 & 0.14 \\
\hline Unidentified $^{\text {b) }}$ & 1.029 & n.d. ${ }^{c)}$ & 0.49 \\
\hline Lathosterol (3) & 1.078 & n.d. ${ }^{c)}$ & 0.92 \\
\hline Unidentified $^{\mathrm{b})}$ & 1.116 & 0.52 & $\operatorname{trace}^{\mathrm{d})}$ \\
\hline 24-Methylenecholesterol (4) & 1.140 & 2.00 & 4.30 \\
\hline Campesterol (5) & 1.156 & 16.35 & 15.62 \\
\hline 24-Methyl-5 $\alpha$ cholest-24(28)-en-3 $\beta$-ol (6) & 1.158 & 0.05 & n.d. ${ }^{c)}$ \\
\hline Campestanol (7) & 1.172 & 7.22 & 5.28 \\
\hline Stigmasterol (8) & 1.199 & 7.58 & 2.34 \\
\hline$\triangle^{22}$-Stigmastenol (9) & 1.219 & 0.80 & 0.26 \\
\hline Unidentified $^{\mathrm{b})}$ & 1.224 & $\operatorname{trace}^{\mathrm{d})}$ & 0.03 \\
\hline Episterol (10) & 1.225 & 0.44 & 1.52 \\
\hline Unidentified $^{\text {b) }}$ & 1.228 & 0.01 & n.d. ${ }^{c)}$ \\
\hline 24-Methyllathosterol (11) & 1.242 & 0.07 & 0.38 \\
\hline Unidentified $^{\mathrm{b})}$ & 1.246 & $\operatorname{trace}^{\mathrm{d})}$ & 0.08 \\
\hline 24-Ethylcholesta-5,25-dien-3 $\beta$-ol (12) & 1.263 & 0.71 & 0.47 \\
\hline Fucosterol (13) & 1.289 & 0.47 & 0.28 \\
\hline Sitosterol (14) & 1.294 & 51.02 & 40.36 \\
\hline Unidentified $^{\mathrm{b})}$ & 1.300 & n.d. ${ }^{c)}$ & 0.01 \\
\hline Isofucosterol (15) & 1.315 & 4.75 & 16.87 \\
\hline Sitostanol (16) & 1.317 & 5.19 & 3.32 \\
\hline Unidentified $^{\text {b) }}$ & 1.323 & n.d. ${ }^{c)}$ & trace $^{\mathrm{d})}$ \\
\hline 24-Ethyl-5 $\alpha$-cholest-24(28)Z-en-3 $\beta$-ol (17) & 1.333 & 0.01 & 0.02 \\
\hline 24-Ethyldesmosterol (18) & 1.354 & 0.65 & 0.57 \\
\hline Unidentified $^{\text {b) }}$ & 1.380 & 0.03 & n.d. ${ }^{c}$ \\
\hline 24-Ethyllathosterol (19) & 1.384 & 0.07 & 0.36 \\
\hline Avenasterol (20) & 1.406 & 0.20 & 1.42 \\
\hline Unidentified $^{\text {b) }}$ & 1.451 & $\operatorname{trace}^{\mathrm{d})}$ & $\operatorname{trace}^{\mathrm{d})}$ \\
\hline
\end{tabular}

a) Relative retention time $\left(\mathrm{Rt}_{\mathrm{R}}\right)$ to the cholesterol TMS ether $(10.645 \mathrm{~min})$.

b) The structure was not determined because of the lack of authenic samples.

c) n.d., not detected.

d) trace, less than $0.01 \%$. 
Table 2 Steroidal 4-En-3-one Composition of the Seeds of Echinochloa frumentacea.

\begin{tabular}{lcc}
\hline \multicolumn{1}{c}{ Steroidal 3-one } & $\mathrm{Rt}_{\mathrm{R}}{ }^{\mathrm{a})}$ & Composition (\%) \\
\hline Steroidal 4-en-3-one fraction (5.4 mg/kg) & & \\
Cholest-4-en-3-one (21) & 1.092 & 0.21 \\
24-Methylcholesta-4,24(28)-dien-3-one (22) & 1.245 & 5.39 \\
24-Methylcholest-4-en-3-one (23) & 1.260 & 21.78 \\
24-Ethylcholesta-4,22E-dien-3-one (24) & 1.310 & 14.19 \\
24-Ethylcholest-4-en-3-one (25) & 1.415 & 46.97 \\
24-Ethylcholesta-4,24(28)Z-dien-3-one (26) & 1.428 & 0.70 \\
Unidentified $^{\text {b) }}$ & 1.457 & 5.80 \\
\hline
\end{tabular}

a) Relative retention time $\left(\mathrm{Rt}_{\mathrm{R}}\right)$ to the cholesterol TMS ether $(10.545 \mathrm{~min})$.

b) The structure was not determined because of the lack of authentic sample.

the free sterol fraction D was fractionated and analyzed. Sterol compositions were calculated from the peak area of total ion chromatogram and the amount of each analyzed sterol fraction. Based on direct GC-MS comparison with authentic samples, sterols listed in Table 1 were identified, in which the compositions of both free and esterified sterol fractions were included. Although the C-24 epimers of 24-methyl- and 24-ethylsterols could not be separated under our GC conditions, we tentatively use trivial names throughout this text.

Among these sterols detected, eight sterols (1, $4,5,7,8,14,15$, and 16) were previously found present in the seeds of Echinochloa crus-galli ${ }^{14)}$. In the current study, cholesterol (1) was present in much higher content in the esterified sterol fraction than the free sterol one. Lathosterol (3) was detected only in the esterified sterol fraction, and episterol (10), 24-methyllathosterol (11), 24ethyllathosterol (19), and avenasterol (20) were found mainly as esterified forms. 24-Methylenecholesterol (4) and isofucosterol (15) were more abundantly detected in the esterified sterol fraction than the free sterol one. Campestanol (7) and sitostanol (16) were accumulated in both free and steryl ester fractions. It is noteworthy that the content of campestanol (7) was higher than that of sitostanol (16) in both sterol fractions, although sitosterol (14) was present in much higher amount than campesterol (5).

Since no report has been published on the steroidal 3-ones in Echinochloa plants, both the steroidal 4-en-3-one fraction B and the $5 \alpha$-steroidal 3-one fraction $\mathrm{C}$ from $E$. frumentacea seeds were examined. Six steroidal 4-en-3-ones listed in the
Table 2 were identified from the fraction B by direct GC-MS comparison with authentic samples. Similarly, the full-scan mass spectral data of 24methyl-5 $\alpha$-cholestan-3-one $\left(27, \mathrm{Rt}_{\mathrm{R}} 1.137\right)$ and 24ethyl-5 $\alpha$-cholestan-3-one (28, $\left.\mathrm{Rt}_{\mathrm{R}} 1.278\right)$ were obtained from the crude fraction C. Although these steroidal 3-ones were reported to occur in many plants ${ }^{4), 6,7), 15,16)}$, the current study is the first report of the co-occurrence of six types of steroidal 4-en-3-ones (21-26) in plants.

The co-occurrence of $\mathrm{C}_{28}$ steroids $(10,4,5,23$, 27 , and 7 ) suggests that $E$. frumentacea may have the same early biosynthetic pathway of episterol (10) $\rightarrow 4 \rightarrow 5 \rightarrow 23 \rightarrow 27 \rightarrow$ campestanol (7) as $A$. thalian $a^{4,16), 17)}$. Based on the present identification data, we propose that all six types of $\Delta^{5}$-sterols, i.e., cholesterol (1), 24-methylenecholesterol (4), isofucosterol (15), campesterol (5), sitosterol (14), and stigmasterol (8), may be converted via the steroidal 4-en-3-ones (21, 22, 26, 23, 25, and 24) into the corresponding C-5 saturated sterols, i.e., cholestanol (2), 24-methyl-5 $\alpha$-cholest-24(28)-en$3 \beta$-ol (6), 24-ethyl-5 $\alpha$-cholest-24(28) $Z$-en-3 $\beta$-ol (17), campestanol (7), sitostanol (16), and $\Delta^{22}$ stigmastenol (9), respectively, by the same series of enzymatic reactions. Metabolic study using deuterio-labeled steroids is necessary to verify our proposals.

(Received Aug. 11, 1999 ; Accepted Nov. 24, 1999)

\section{References}

1) M. Sugano, Nippon Nogeikagaku Kaishi (review in Japanese), 71, 769 (1997).

2) H. Hibino, J. Jpn. Oil Chem. Soc. (review in Japanese), 46, 1127 (1997).

3) A. Sakurai, "Brassinosteroids," ed. by A. Sakurai, 
T. Yokota, S.D. Clouse, Springer-Verlag, Tokyo, (1999) p. 91.

4) T. Noguchi, S. Fujioka, S. Takatsuto, A. Sakurai, S. Yoshida, J. Li, J. Chory, Plant Physiol., 120, 833 (1999).

5) S. Takatsuto, T. Kawashima, T. Noguchi, S. Fujioka, A. Sakurai, J. Jpn. Oil Chem. Soc., 46, 1499 (1997).

6) S. Takatsuto, T. Kawashima, J. Jpn. Oil Chem. Soc., 47, 783 (1998).

7) S. Takatsuto, N. Kosuga, B. Abe, T. Noguchi, S. Fujioka, T. Yokota, J. Plant. Res., 112, 27 (1999).

8) B. Abe, S. Takatsuto, J. Jpn. Oil Chem. Soc., 48, 41 (1999).

9) B. Abe, E. Itokawa, S. Takatsuto, Nippon Nogeikagaku Kaishi (in Japanese), 73, 419 (1999).

10) S. Takatsuto, C. Gotoh, T. Noguchi, S. Fujioka, $J$. Jpn. Oil Chem. Soc., 48, 37 (1999).

11) S. Takatsuto, E. Tsunokawa, T. Noguchi, S.
Fujioka, J. Jpn. Oil Chem. Soc., 48, 347 (1999).

12) Y. Narumi, T. Watanabe, T. Noguchi, B. Abe, S. Fujioka, T. Yokota, S. Takatsuto, J. Jpn. Oil Chem. Soc., 48, 1307 (1999).

13) Y. Narumi, C. Gotoh, S. Takatsuto, unpublished work.

14) S. Takatsuto, T. Kawashima, J. Jpn. Oil Chem. Soc., 47, 187 (1998).

15) T. Akihisa, W.C.M.C. Kokke, T. Tamura, "Physiology and Biochemistry of Sterols," ed. by G.W. Patterson, W.D. Nes, American Oil Chemists' Society, Champaign, Illinois, (1991) p. 172.

16) U. Klahre, T. Noguchi, S. Fujioka, S. Takatsuto, T. Yokota, T. Nomura, S. Yoshida, N.-H. Chua, Plant Cell, 10, 1677 (1998).

17) S. Choe, T. Noguchi, S. Fujioka, S. Takatsuto, C.P. Tissier, B.D. Gregory, A.S. Ross, A. Tanaka, S. Yoshida, F.E. Tax, K.A. Feldmann, Plant Cell, 11, 207 (1999). 


\section{[ノート］ヒエ種子中に含まれるステロールおよび \\ ステロイド系3-オンの同定}

鳴海安久 ${ }^{* 1}$. 後藤千春*1 . 野口貴弘 ${ }^{* 2}$

藤岡昭三*3 - 横田孝雄*4 ・高津戸秀*1

*1 上越教育大学自然系化学教室（ $\bar{\top} 943-8512$ 新潟県上越市山屋敷町 1 )

*2 夕マ生化学 († 163-0704 東京都新宿区西新宿 2-7-1)

*3 理化学研究所植物機能研究室 (T 351-0198 埼玉県和光市広沢 2-1)

*4 帝京大学バイオサイエンス学科（テ 320-8551 栃木県宇都宮市豊郷台 1-1）

ヒエ (Echinochloa frumentacea (Roxb.) Link) 種子中に含まれるステロールおよびステロイド系 3-オンの同 定を行った。20 種類のステロールを標品との GC-MSによる直接比較により同定し, 遊離型およびエステル型ス テロール分画のステロール組成を解明した。さらに，ステロイド系3-オンとして 8 種の化合物を同定した。

（連絡者：高津戸 秀） Vol.49, No.4，367（2000）

\section{[速報］フォスファチジルコリン/セラミド 3 混合単分子膜}

\section{および混合二分子膜中でのドメイン形成と相分離}

井村知弘 ${ }^{* 1}$ - 酒井秀樹*1,*2 - 山内仁史 ${ }^{* 1}$. 貝瀬千尋*1

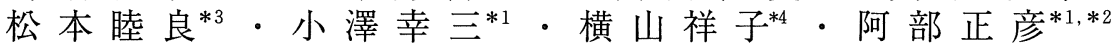

* 1 東京理科大学理工学部（干 278-8510 千葉県野田市山崎 2641）

* 2 東京理科大学界面科学研究所（广 162-0825 東京都新宿区神楽坂 1-3）

* 3 物質工学工業技術研究所（† 305-8565 茨城県つくば市東 1-1）

* 4 共立薬科大学（干 105-8512 東京都港区芝公園 1-5-30)

L- $\alpha$ - ジパルミトイルフォスファチジルコリン (DPPC) と $2 S, 3 S, 4 R-2-$ ステアロイルアミド $-1,3,4-$ オタ デカントリオール (Ceramide 3) から成る混合単分子膜中および混合二分子膜中における相互作用を表面圧測定, 原子間力顕微法 (AFM) および示差走査熱量測定（DSC）により検討した。

表面圧 $10 \mathrm{mN} / \mathrm{m}$ でマイカ上に一層に累積した DPPC/Ceramide 3 混合単分子膜（LB 膜）の AFM 画像には楕円 形のドメインが示された。DPPCリポソーム溶液を用いて測定した DSC 曲線からは，DPPC二分子膜の相転移 温度が Ceramide 3 の添加によって高温側にシフトすること，および DPPC 単独系で見られたシャープなピーク は DPPC/Ceramide 3 混合系ではブロードなピークに変化し，更には Ceramide 3 のモル分率が高いものでは DSC ピークが分裂することが観察された。結論として，DPPC/Ceramide 3 混合単分子膜中および混合二分子膜中に おいて相分離が認められた。Ceramide 3 は液体膨張膜の状態にある DPPC と相互作用を起こすことによって, ドメイン形成を伴なう相分離を起こすことが示唆された。 